\title{
DESEMPENHO COGNITIVO-LINGUÍSTICO DE ESCOLARES COM DISTÚRBIO DE APRENDIZAGEM ${ }^{1}$
}

\author{
Cláudia Silva* \\ Simone Aparecida Capellini ${ }^{\#}$
}

\begin{abstract}
RESUMO. O estudo tem como objetivo caracterizar e comparar o desempenho cognitivo-linguístico de escolares com distúrbio de aprendizagem com escolares com bom desempenho acadêmico. Participaram 40 escolares de 8 a 12 anos de idade, de $2^{\mathrm{a}}$ a $4^{\mathrm{a}}$ séries do Ensino Fundamental de escolas municipais da cidade de Marília - SP, divididos em GI (escolares com bom desempenho acadêmico) e GII (escolares com distúrbio de aprendizagem). Como procedimento foi utilizado o Teste de Desempenho Cognitivo-Linguístico, versões coletiva e individual. Os resultados evidenciaram desempenho superior do GI em relação ao GII nas habilidades de leitura, escrita, velocidade de processamento, processamento auditivo e visual. Concluiu-se que o desempenho inferior do GII nas habilidades indica uma limitação no desempenho linguístico desses escolares se comparados com os do GI, exceto na habilidade de consciência fonológica, em que, os grupos apresentaram dificuldades semelhantes, sugerindo que essa dificuldade não seja específica de escolares com distúrbio de aprendizagem.
\end{abstract}

Palavras-chave: Aprendizagem; avaliação; leitura.

\section{COGNITIVE-LINGUISTIC PERFORMANCE OF STUDENTS WITH LEARNING DISABILITIES}

\begin{abstract}
The study aims to characterize and to compare the cognitive-linguistic performance of students with learning disabilities with good academic performance. Forty students participated in this study, aged between 8 to 12 years old, from $2^{\text {nd }}$ to $4^{\text {th }}$ grades of elementary education in municipal schools in Marília-SP, divided into GI (good academic performance students) and GII (students with learning disabilities). As procedure we used the Test Cognitive-Linguistic Performance - collective and individual version. The results evidenced better performance of GI in relation to GII in skills of reading, writing, auditory processing, visual processing and processing speed. Conclusion: The lower performance of GII in skill indicates a limitation in the linguistic performance of these students compared to good academic performances, except in skill of phonological awareness, in which, the groups showed similar difficulties, suggesting that this difficulty is not specific to students with learning disabilities..
\end{abstract}

Key words: Learning; evaluation; reading.

\section{RENDIMIENTO COGNITIVO-LINGÜÍSTICO DE ESTUDIANTES CON TRASTORNOS DE APRENDIZAJE}

RESUMEN. El estudio tiene como objetivo caracterizar y comparar el rendimiento cognitivo-lingüístico de estudiantes con trastornos de aprendizaje con estudiantes con buen rendimiento académico. Los participantes fueron 40 niños escolares de 8 a 12 años de edad, de $2^{\circ}$ a $4^{\circ}$ grados de la escuela primaria de la ciudad de Marília-SP. Fueron divididos en GI (estudiantes con buen rendimiento académico) y GII (estudiantes con trastornos de aprendizaje). El procedimiento utilizado fue la prueba de rendimiento cognitivo-lingüístico, colectivo e individual. Los resultados sugieren desempeño superior del GI si comparado con GII en las habilidades de lectura, escrita, velocidad de procesamiento, procesamiento auditivo y visual. Conclusión: el rendimiento inferior de GII en las habilidades apuntando a una limitación en la actuación lingüística de los estudiantes frente al GI, excepto en la habilidad de consciencia fonológica, en que, los grupos tienen dificultades similares, lo que sugiere que esta dificultad no es específica de estudiantes con trastornos de aprendizaje.

Palabras-clave: Aprendizaje; evaluación; lectura.

1 Apoio: Capes.

Fonoaudióloga. Doutoranda em educação pelo programa de Pós-graduação da Faculdade de Filosofia e Ciências da Unesp/Marília-SP, Brasil.

\# Fonoaudióloga. Doutora em Ciências Médicas - Faculdade de Ciências Médicas da Universidade Estadual de Campinas - FCM/UNICAMPCampinas - SP. Professora do Departamento de Fonoaudiologia e do Programa de Pós-Graduação em Educação da Faculdade de Filosofia e Ciências da Universidade Estadual Paulista - FFC/UNESPk, Marília-SP, Brasil. 
Segundo Salles e Parente (2007), a leitura e a escrita são atividades complexas, constituídas de múltiplos processos interdependentes. A Psicologia Cognitiva e a Neuropsicologia Cognitiva focalizam a análise dos processos neurocognitivos subjacentes às habilidades de ler e escrever, tanto no leitor/escritor proficiente como no leitor/escritor iniciante e dos distúrbios de leitura e escrita.

De acordo com a literatura, para que ocorra o desenvolvimento e a aquisição da leitura e da escrita é necessário o acesso ao léxico mental, à memória de trabalho e à consciência fonológica, as quais, quando alteradas, comprometem diretamente o domínio do sistema de escrita nos níveis ortográfico e fonológico (Cardoso-Martins \& Pennington, 2001; Barrera \& Maluf, 2003; Cardoso-Martins \& Pennington, 2004; Gray \& McCutchen, 2006; Mousinho \& Correa, 2009).

Entre as alterações neuropsicológicas e neurocognitivas que podem acarretar atraso na aquisição de habilidades e no desenvolvimento escolar encontra-se o distúrbio de aprendizagem (Ciasca, Capellini, \& Tonelotto, 2004).

O distúrbio de aprendizagem é caracterizado pela disfunção neurológica ou hereditária, que é responsável pela alteração do processamento cognitivo e da linguagem, caracterizada por um funcionamento cerebral atípico. Como consequência dessa disfunção, a forma como crianças com distúrbio de aprendizagem processam e adquirem informações é diferente do funcionamento típico de crianças sem dificuldade na fase de aprendizagem escolar. Sendo assim, o distúrbio de aprendizagem pode se manifestar nas áreas acadêmicas que necessitam de decodificação ou identificação de palavras, como a leitura, compreensão de leitura, raciocínio matemático, atividades de soletração e escrita de palavras e textos (Silver, Ruff, Iverson, Barth, Broshek, Bush, et.al. 2008; Wu, Huang \& Meng, 2008).

Segundo Sideridis (2007), os fatores pedagógicos, psicológicos, socioeconômicos e culturais estão implícitos, sendo considerados fatores agravantes nas manifestações comportamentais e de linguagem do distúrbio de aprendizagem. Desta forma, segundo o autor, o distúrbio de aprendizagem é uma condição que se prolonga por toda a vida, e o impacto da dificuldade no desenvolvimento do individuo vai depender de fatores sociais, emocionais e educacionais e das funções ocupacionais, que por sua vez dependem das circunstâncias diárias, das relações interpessoais, do domínio de habilidades e de dificuldades individuais (Vogel, Fresko \& Wertheinm, 2007; Silver et al., 2008).
Os estudos realizados nos últimos 20 anos têm demonstrado que as manifestações do distúrbio de aprendizagem no escolar ocorrem por falhas no processamento cognitivo, linguístico, auditivo e visual, e que, em decorrência dessas falhas, altera-se o acionamento de mecanismos cognitivos para analisar, sintetizar, manipular, estocar e evocar informações linguísticas, prejudicando a aprendizagem de sistemas de escrita com base alfabética (Capovilla, Capovilla, \& Suiter, 2004; Heiman \& Kariv, 2004; Paula, Mota $\&$ Keske-Soares, 2005; Silver et al., 2008).

Com base no exposto acima, este estudo tem por objetivo caracterizar o desempenho cognitivolinguístico de um grupo de escolares com distúrbio de aprendizagem em comparação com o desempenho de um grupo de escolares com bom desempenho acadêmico.

\section{MÉTODO}

Este estudo transversal foi realizado após a aprovação do Comitê de Ética em Pesquisa da Faculdade de Filosofia e Ciências da Universidade Estadual Paulista - CEP/FFC/UNESP sob o protocolo n. ${ }^{\circ} 2596 / 2007$. Dele participaram 40 escolares de $2^{\mathrm{a}}$ a $4^{\text {a }}$ séries do Ensino Fundamental de escolas municipais de Marília, sendo 35 (87,5\%) do gênero masculino e $5(12,5 \%)$ do gênero feminino, com idade entre 8 e 12 anos, distribuídos em dois grupos: grupo I (GI), composto por 20 escolares com bom desempenho acadêmico, dos quais $16(80 \%)$ eram do gênero masculino e 4 (20\%) do gênero feminino; e grupo II (GII), formado por 20 escolares com diagnóstico interdisciplinar de aprendizagem, dos quais 19 eram do gênero masculino e 1 (um) do gênero feminino.

Os escolares com bom desempenho acadêmico foram selecionados pelos professores seguindo o critério de desempenho satisfatório (média de nota variando entre 7,0 e 8,0 ) em dois bimestres consecutivos em avaliação de leitura e escrita.

O diagnóstico de distúrbio de aprendizagem foi realizado por uma equipe interdisciplinar do Centro de Estudos da Educação e Saúde - CEES/UNESP Marília e Ambulatório de Neurologia Infantil Aprendizagem do Hospital das Clínicas da Faculdade de Medicina da Unesp - Botucatu, incluindo avaliação fonoaudiológica, neurológica, pedagógica, neuropsicológica e de neuroimagem (Single Photon Emission Computed Tomograpy - SPECT).

Usou-se como critério de exclusão a criança não apresentar deficiência sensorial, motora ou cognitiva, e como critério de inclusão, a criança não ter queixa 
auditiva ou visual descrita nos prontuários escolares e seus pais ou responsáveis assinarem o Termo de Consentimento Livre e Esclarecido.

Neste estudo foram realizados o Teste de Desempenho Cognitivo-Linguístico - versão coletiva - e o Teste de Desempenho Cognitivo-linguístico versão individual.

No primeiro dos dois testes foi aplicada a versão coletiva do Teste (Silva, Capellini, Gonzaga, Galhardo, Cruvinel, \& Smythe, 2007; Capellini \& Smythe, 2008) em todos os escolares deste estudo. Esta versão é composta dos seguintes subtestes:

- Reconhecimento do alfabeto em sequência, em que o escolar deveria escrever em sequência todas as letras do alfabeto;

- Cópia de formas, em que o escolar deveria copiar quatro diferentes formas geométricas;

- Ditado de 30 palavras reais e 10 palavras inventadas, em que o examinador pronunciou um total de 40 palavras que o escolar deveria escrever;

- Memória de curta duração, em que o escolar, após ouvir o examinador pronunciar 14 sequências de dígitos, com dois a nove dígitos cada, devia escrever todos os dígitos

No Teste de Desempenho Cognitivo - Linguístico - versão individual foi aplicada a versão individual do Teste (Silva et al., 2007; Capellini \& Smythe, 2008) em todos os escolares deste estudo. Esta versão se constitui dos seguintes subtestes:

- Leitura de palavras, em que o escolar realizava em voz alta a leitura de 70 palavras reais, enquanto o examinador anotava as palavras lidas corretamente em 1 (um) minuto;

- Leitura de não palavras, em que o escolar lia em voz alta 10 falsas palavras, enquanto o examinador marcava o tempo gasto na leitura e as palavras lidas corretamente;

- Consciência fonológica: esse teste se compôs de 10 subtestes de aliteração e 20 subtestes de rima, sendo o escolar orientado pelo examinador a ouvir uma sequência de três palavras e escolher duas delas que apresentassem aliteração ou rima;

- Processamento auditivo: teste composto de subtestes em que o escolar devia fazer a discriminação de sons, repetição de números, repetição inversa de números, repetição de palavras e repetição de falsas palavras, e o examinador determinava as atividades a serem realizadas em voz alta e o escolar as realizava também em voz alta;

- Processamento visual: teste composto de subtestes de memória visual com oito sequências de cartões, compostas de dois a oito cartões cada, em que o examinador apresentava uma sequência por vez para o escolar, este observava a sequência e em seguida deveria reproduzi-la com os cartões apresentados pelo examinador;

- Velocidade de processamento: teste composto de dois subtestes, sendo um constituído de um quadro com quatro figuras diferentes que se repetiam em ordem aleatória, e o outro, de números de zero a nove também, apresentados aos escolares em ordem aleatória; nos dois subtestes o escolar nomeava rapidamente as figuras e os números apresentados pelo examinador.

A coleta de dados foi realizada no Centro de Estudos da Educação e Saúde - CEES/UNESPMarília em período contrário ao de estudos dos escolares.

Os resultados foram analisados estatisticamente com nível de significância de $5 \%(0,050)$ para a aplicação dos testes estatísticos, utilizando-se o programa SPSS (Statistical Package for Social Sciences), em sua versão 13.0. O teste utilizado para análise estatística foi o Teste de Mann-Whitney, com o intuito de verificar possíveis diferenças entre as médias dos grupos formados. Os resultados estatisticamente significantes foram marcados com um $\operatorname{asterisco}(*)$.

\section{RESULTADOS}

A apresentação dos resultados do Teste de Desempenho Cognitivo-Linguístico individual e coletivo será realizada por meio de tabelas, de acordo com as habilidades de leitura, de escrita, de consciência fonológica, de processamento auditivo, de processamento visual e de velocidade de processamento.

- Quanto à habilidade de leitura, serão descritos o desempenho dos grupos nas provas de reconhecimento do alfabeto, leitura de palavras, leitura em 1 (um) minuto de falsas palavras e de palavras existentes.

- Quanto à habilidade de escrita, será descrito o desempenho dos grupos nas provas de ditado de palavras, ditado de falsas palavras e ditado total; 
- Quanto à habilidade de consciência fonológica, será descrito o desempenho dos grupos nas provas de aliteração e rima;

- Quanto à habilidade de processamento auditivo, será descrito o desempenho dos grupos nas provas de discriminação de sons, repetição de palavras, repetição de falsas palavras, sequência de números e sequência de números invertidos.

- Quanto à habilidade de processamento visual, será descrito o desempenho dos grupos nas provas de cópia de formas, memória visual com dois cartões, memória visual com três cartões, memória visual com quatro cartões e memória visual com cinco cartões.

- Quanto à habilidade de velocidade de processamento, será descrito o desempenho dos grupos nas provas de nomeação de figuras e leitura de números.

Na comparação entre o desempenho do grupo GI e o do GII, foi possível verificar na habilidade de leitura diferença estatisticamente significante para os escolares do GI nos subtestes de alfabeto, leitura de palavras e leitura de falsas palavras, e diferença estatisticamente significante para GII no subteste de leitura de palavras corretas em um minuto. Isso indica que os escolares do GI apresentaram desempenho superior em termos de decodificação fonológica e acesso ao léxico, em relação à leitura de falsas palavras e à de palavras existentes, respectivamente ( tabela 1).

$\mathrm{Na}$ habilidade de escrita os escolares do GI apresentaram desempenho superior ao dos escolares do GII nos subtestes de ditado de palavras e ditado total, o que sugere melhor domínio desses escolares quanto à memória lexical. O GI e GII apresentaram desempenhos semelhantes quando comparados seus desempenhos no subteste de ditado de falsas palavras, o que sugere dificuldade de ambos os grupos em acessar a memória fonológica (tabela 1).

Ao comparar os resultados de GI e GII nos subtestes de rima e aliteração e da habilidade de consciência fonológica, foi possível verificar que ambos os grupos não apresentaram diferença estatisticamente significante, o que sugere dificuldade em desempenhar atividades que envolvam, diretamente, habilidades fonológicas (Tabela 1).

Tabela 1 - Distribuição da média, desvio-padrão e valor de p do desempenho dos escolares dos GI e GII na habilidade de Leitura, Escrita e Consciência Fonológica do Teste de Desempenho Cognitivo - Linguístico.

\begin{tabular}{|c|c|c|c|c|c|}
\hline Habilidades do Teste & Variáveis & Grupo & Média & Desvio-padrão & Significância (p) \\
\hline \multirow{8}{*}{ Leitura } & Alf & GI & 26,00 & 0,00 & \multirow{2}{*}{$0,041^{*}$} \\
\hline & Alf & GII & 24,00 & 2,21 & \\
\hline & LP & GI & 231,80 & 93,03 & \multirow{2}{*}{$0,007 *$} \\
\hline & LP & GII & 164,20 & 91,21 & \\
\hline & LNP & GI & 18,90 & 5,70 & \multirow{2}{*}{$0,011^{*}$} \\
\hline & LNP & GII & 13,70 & 2,75 & \\
\hline & Corlm & GI & 26,30 & 10,69 & \multirow{2}{*}{$0,005^{*}$} \\
\hline & Cor1m & GII & 36,20 & 9,57 & \\
\hline \multirow{6}{*}{ Escrita } & Dit_P & GI & 26,00 & 1,15 & \multirow{2}{*}{$0,008 *$} \\
\hline & Dit_P & GII & 24,40 & 1,17 & \\
\hline & Dit_NP & GI & 7,40 & 1,17 & \multirow{2}{*}{0,136} \\
\hline & Dit_NP & GII & 6,60 & 0,97 & \\
\hline & Dit_T & GI & 33,40 & 1,65 & \multirow{2}{*}{$0,015^{*}$} \\
\hline & Dit_T & GII & 31,00 & 1,94 & \\
\hline \multirow{4}{*}{$\begin{array}{l}\text { Consciência } \\
\text { Fonológica }\end{array}$} & Alit & GI & 9,80 & 0,42 & \multirow{2}{*}{0,914} \\
\hline & Alit & GII & 9,70 & 0,42 & \\
\hline & Rima & GI & 19,70 & 0,95 & \multirow{2}{*}{0,584} \\
\hline & Rima & GII & 19,60 & 0,97 & \\
\hline
\end{tabular}

Legenda: Alf : alfabeto, LP : leitura de palavras, LNP: leitura de não palavras, Cor1m: corretas em 1 minuto, Dit_P: ditado de palavras, Dit_NP: ditado de não palavras, Dit_T: ditado total, Alit: aliteração.

$\mathrm{Na}$ habilidade de processamento auditivo os escolares do GI apresentaram desempenho estatisticamente significante nos subtestes de discriminação de sons, repetição de falsas palavras e números invertidos, se comparado ao desempenho do GII, o que sugere desempenho superior do GI nas atividades que envolvem memória sequencial auditiva e de curto prazo (tabela 2). 
Em relação à habilidade de processamento visual, foi possível verificar que os escolares do GI apresentaram diferença estatisticamente significante nos subtestes de cópia de formas, memória visual para dois cartões e memória visual para três cartões, se comparados com os escolares de GII, o que sugere desempenho superior em memória de sequenciamento visual para os escolares do GI (tabela 2).
Ao compararmos o desempenho dos escolares do GI com o do GII na habilidade de velocidade de processamento, foi possível verificar que os escolares do GI apresentaram desempenho superior nos subtestes de nomeação de figuras e leitura de números, resultados que equivalem ao tempo gasto, em segundos, em cada atividade (tabela 2).

Tabela 2 - Distribuição da média, desvio-padrão e valor de p do desempenho dos escolares dos GI e GII na habilidade de Processamento Auditivo, Processamento Visual e Velocidade de Processamento do Teste de Desempenho Cognitivo - Linguístico.

\begin{tabular}{|c|c|c|c|c|c|}
\hline Habilidades do Teste & Variáveis & Grupo & Média & Desvio-padrão & Significância (p) \\
\hline \multirow{10}{*}{$\begin{array}{c}\text { Processamento } \\
\text { Auditivo }\end{array}$} & DS & GI & 19,60 & 0,97 & \multirow{2}{*}{$0,043 *$} \\
\hline & DS & GII & 17,30 & 4,16 & \\
\hline & RepP & GI & 4,30 & 0,95 & \multirow{2}{*}{0,063} \\
\hline & RepP & GII & 3,70 & 1,49 & \\
\hline & RepNP & GI & 300,00 & 40,00 & \multirow{2}{*}{$0,014 *$} \\
\hline & RepNP & GII & 264,00 & 50,60 & \\
\hline & Núm & GI & 396,00 & 127,12 & \multirow{2}{*}{0,314} \\
\hline & Núm & GII & 372,00 & 73,76 & \\
\hline & N_Inv & GI & 252,00 & 83,90 & \multirow{2}{*}{$0,031^{*}$} \\
\hline & N_Inv & GII & 192,00 & 47,33 & \\
\hline \multirow{10}{*}{$\begin{array}{c}\text { Processamento } \\
\text { Visual }\end{array}$} & $\mathrm{CP}$ & GI & 12,90 & 4,46 & \multirow{2}{*}{$0,011 *$} \\
\hline & $\mathrm{CP}$ & GII & 8,30 & 4,22 & \\
\hline & MV2 & GI & 4,00 & 0,00 & \multirow{2}{*}{$0,009 *$} \\
\hline & MV2 & GII & 2,90 & 0,74 & \\
\hline & MV3 & GI & 4,80 & 0,79 & \multirow{2}{*}{$0,020 *$} \\
\hline & MV3 & GII & 3,80 & 1,03 & \\
\hline & MV4 & GI & 5,40 & 1,07 & \multirow{2}{*}{0,809} \\
\hline & MV4 & GII & 5,30 & 1,57 & \\
\hline & MV5 & GI & 6,90 & 1,20 & \multirow{2}{*}{0,131} \\
\hline & MV5 & GII & 6,30 & 1,70 & \\
\hline Velocidade & Nfig_T & GI & 40,10 & 7,89 & \multirow{2}{*}{$0,008^{*}$} \\
\hline De & Nfig_T & GII & 32,50 & 7,68 & \\
\hline \multirow[t]{2}{*}{ Processamento } & NN_T & GI & 39,70 & 7,75 & \multirow{2}{*}{$0,016^{*}$} \\
\hline & NN_T & GII & 33,70 & 6,20 & \\
\hline
\end{tabular}

Legenda: DS: discriminação de sons, RepP: repetição de palavras, RepNP: repetição de não palavras, Núm: sequência de números, N_Inv: sequência de números invertidos, CP: cópia de formas, MV2: memória visual com 2 cartões, MV3: memória visual com 3 cartões, MV4: memória visual com 4 cartões, MV5: memória visual com 5 cartões, Nfig_T: nomeação de figuras tempo, NN_T: nomeação de números tempo.

\section{DISCUSSÃO}

O presente estudo buscou caracterizar e comparar o desempenho cognitivo-linguístico de escolares com distúrbio de aprendizagem com o de escolares com bom desempenho acadêmico nas habilidades do Teste de Desempenho Cognitivo-Linguístico.
Os resultados revelaram que os escolares do GII apresentaram desempenhos semelhantes aos dos escolares do GI no que se refere às provas de rima e aliteração e da habilidade de consciência fonológica, sugerindo que tais dificuldades não podem ser consideradas características apenas de escolares com distúrbio de aprendizagem, pois, quando o princípio 
alfabético não é inserido como instrução formal no contexto da alfabetização, podem ocorrer dificuldades na percepção fonológica tanto de escolares com distúrbio de aprendizagem como daqueles com bom desempenho acadêmico (Harn, Stoolmiller \& Chard, 2008; Thomson \& Goswami, 2008; Murphy \& Schochat, 2009).

O desempenho inferior dos escolares do GII em relação à habilidade de leitura para as provas de alfabeto, leitura de palavras, leitura de falsas palavras e número de palavras lidas corretamente em um minuto, e em relação à habilidade de escrita, nas provas de ditado de palavras e ditado total, permitenos destacar que estes escolares com distúrbio de aprendizagem apresentam dificuldade na precisão da leitura e na decodificação fonológica tanto de palavras reais como de falsas palavras, o que pode limitar o desempenho linguístico, representando dificuldades aos escolares quanto ao uso de palavras novas. Nossos resultados são compatíveis com a literatura recente, que sugere que o domínio da leitura e da escrita é influenciado pelo déficit no processamento cognitivolinguístico, caracterizando escolares com diagnóstico de distúrbio de aprendizagem (Capovilla et al., 2004; Vloedgraven \& Verhoeven, 2007; Ryder, Tunmer \& Greaney, 2008; Araújo \& Minervino, 2008; Mousinho \& Correa, 2009).

Conforme descrito na literatura, na realização de provas que envolvem habilidades de processamento auditivo, processamento visual e velocidade de processamento estão envolvidos componentes como a memória fonológica de trabalho, acesso ao léxico mental, organização e processamento da informação visual e auditiva. A dificuldade em acessar esses componentes foi evidenciada nos escolares do GII deste estudo, os quais apresentaram desempenho inferior nas atividades de discriminação de sons, repetição de falsas palavras e sequência de números invertidos, na habilidade de processamento auditivo, na cópia de formas, memória visual com dois e três cartões, na habilidade de processamento visual e principalmente nas provas da habilidade de velocidade de processamento, que são de nomeação de figuras e nomeação de números (Rodrigues \& Befi-Lopes, 2009; Vaessen, Gerretsen \& Blomert, 2009).

O desempenho dos escolares com distúrbio de aprendizagem nas habilidades de processamento auditivo, processamento visual e velocidade de processamento sugere alteração da memória de trabalho no desempenho das atividades que envolvem retenção e manipulação de informações. De acordo com a literatura, a memória de trabalho e o acesso fonológico ao léxico mental ocorrem de forma conjunta e inter-relacionada, de modo que, ao ocorrerem alterações no desenvolvimento desses componentes o desenvolvimento cognitivo-linguístico também será alterado (Ávila, 2004; Cunha \& Capellini, 2009).

\section{CONCLUSÃO}

De acordo com os resultados obtidos neste estudo, podemos concluir que o desempenho cognitivolinguístico de escolares com distúrbio de aprendizagem nas habilidades de leitura, escrita, processamento auditivo, processamento visual e velocidade de processamento apresenta-se alterado, apontando para uma limitação no desempenho linguístico desses escolares em comparação com os escolares com bom desempenho acadêmico. No que se refere à habilidade de consciência fonológica, os escolares com distúrbio de aprendizagem apresentaram dificuldades semelhantes às mostradas pelo grupo de escolares com bom desempenho acadêmico, o que sugere que essa dificuldade não é específica de escolares com esse quadro.

\section{REFERÊNCIAS}

Araújo, M. R., \& Minervino, C. A. S. M. (2008). Avaliação cognitiva: leitura, escrita e habilidades relacionadas. Psicologia em Estudo, 4(13), 859-865.

Ávila, C. R. B. (2004). Tratado de Fonoaudiologia. In L. P. Ferreira, L. P., D. M. Befi-Lopes; S. C. O Limongi (Org.), Consciência fonológica (pp. 815-824). São Paulo: Roca.

Barrera, S. D., \& Maluf, M. R. (2003). Consciência metalinguística e alfabetização: um estudo com crianças da primeira série do ensino fundamental. Psicologia, Reflexão e Critica, 16(3), 491-502.

Capellini, A. S., \& Smythe, I. (2008). Protocolo de Avaliação de Habilidades Cognitivo - Linguísticas. Livro do Profissional e do Professor. Marília: Fundepe.

Capovilla, A. G. S., Capovilla, F. C., \& Suiter, I. (2004). Processamento cognitivo em crianças com e sem dificuldades de leitura. Psicologia em Estudo, 3(9), 449-458.

Cardoso-Martins, C., \& Pennington, B. B. (2001). Qual a contribuição da nomeação seriada rápida para a habilidade de leitura e escrita? Evidências de crianças e adolescentes com e sem dificuldades de leitura. Psicologia: Reflexão e Crítica, 14(2), 387-397.

Cardoso-Martins, C., \& Pennington, B. B. (2004). The relationship between phoneme awareness and rapid naming skills and literacy acquisition: the role of development period and reading ability. Scient Stud Read, 8(1), 27-52.

Ciasca, S. M., Capellini, S. A., \& Tonelotto, J. M. F. (2004). Distúrbios específicos de aprendizagem. In S. M. Ciasca. (Org.), Distúrbios de aprendizagem: proposta de avaliação interdisciplinar (pp. 55-66). São Paulo: Casa do Psicólogo. 
Cunha, V. L. O., \& Capellini, S. A. (2009). Desempenho de escolares de $1^{\mathrm{a}}$ a $4^{\mathrm{a}}$ série do ensino fundamental nas provas de habilidades metafonológicas e de leitura - PROHMELE. Revista da Sociedade Brasileira de Fonoaudiologia, 14(1), 56-68.

Gray, A., \& Mccutchen, D. (2006) Young readers' use of phonological information: phonological awareness, memory, and comprehension. Journal of Learning Disabilities, 39(4), 325-333.

Harn, B. A., Stoolmiller, M., \& Chard, D. J. (2008). Measuring the dimensions of alphabetic principle on the reading development of first graders. The role of automaticity and unitization. Journal of Learning Disabilities, 41(2), 143-157.

Heiman, T. \& Kariv, D. (2004). Manifestations of learning disabilities in university students. Implications for coping and adjustment. Education, 125, 313-325.

Mousinho, R., \& Correa, J. (2009). Habilidades linguístico cognitivas em leitores e não-leitores. Pró-Fono Revista de Atualização Científica, 21(2), 113-118.

Murphy, C. F. B., \& Schochat, E. (2009). Correlações entre leitura, consciência fonológica e processamento temporal auditivo. PróFono Revista de Atualização Científica, 21(1), 13-18.

Paula, G. R., Mota, H. B, \& Keske-Soares, M. A. (2005). A terapia em consciência fonológica no processo de alfabetização. Pró-Fono: Revista de Atualização Científica, 17(2), 175-184.

Rodrigues, A., \& Befi-Lopes, D. M. (2009). Memória operacional fonológica e suas relações com o desenvolvimento da linguagem infantil. Pró-Fono: Revista de Atualização Científica, 21(1), 6368.

Ryder J. F., Tunmer, W. E., \& Greaney, K. T. (2008). Explicit instruction in phonemic awareness and phonemically based decoding skills as an intervention strategy for struggling readers in whole language classrooms. Springer Science and Business Media B. V., 21, 349-369.

Salles, J. F., \& Parente, M. A. P. (2007). Avaliação da leitura e escrita de palavras em crianças de $2^{\mathrm{a}}$ série: abordagem neuropsicológica cognitiva. Psicologia: Reflexão e Critica, 20(2), 220-228.

Sideridis, G. D. (2007). Why Students With LD Depressed? A Goal Orientation Model of Depression Vulnerability. Journal of Learning Disabilities, 40(6), 526-539.
Silva, C., Capellini, S. A., Gonzaga, J., Galhardo, M. T., Cruvinel, P., \& Smythe, I. (2007) Desempenho cognitivo - linguístico de escolares de $1^{\mathrm{a}}$ a $4^{\mathrm{a}}$ série do ensino público municipal. Revista da Associação Brasileira de Psicopedagogia, 24(73), 30-44.

Silver, C. H., Ruff, R. M., Iverson, G. L., Barth, J. T., Broshek, D. K., Bush, S. S., et.al. (2008). Nan Policy and Planning Commitee. Learning disabilities: The need for neuropsycological evaluation. Archives of Clinical Neuropsychology, 23, 217-219.

Thomson, J. M., \& Goswami, U. (2008). Rhythmic processing in children with developmental dyslexia: auditory and motor rhythms link to reading and spelling. Journal Physiology Paris, 102(1-3), 120-129.

Vaessen, A., Gerretsen, P., \& Blomert, L. (2009). Naming problems do not reflect a second independent core deficit in dyslexia: double deficits explored. Jounal of Experimental Child Psychology, 102, 202-221.

Vloedgraven, J. M. T., \& Verhoeven, L. (2007). Screening of phonological awareness in the early elementary grades: an IRT approach. The International Dyslexia Association, 57, 33-50.

Vogel, G., Fresko, B., \& Wertheim, C. (2007). Peer Tutoring for College Students With Learning Disabilities: Perceptions of Tutors and Tutees. Journal of Learning Disabilities, 40(6), 485493.

Wu, T. K., Huang, S. C., \& Meng, Y. R. (2008). Evaluation of ANN and SVM classifiers as predictors to the diagnosis of students with learning disabilities. Expert Systems with Appplications, 34, 846-856. 